\title{
Correction to: Processability of Polymeric Composites
}

\section{Correction to:}

A. K. Ghosh, M. Dwivedi, Processability of Polymeric Composites, https://doi. org/10.1007/978-81-322-3933-8

The below listed late corrections have been carried out in the following chapters of the current version:

1. Chapter 1, p. 6, under heading Answer, symbol ' $\delta$ ' was replaced by ' $M$ '

2. Chapter 3, p. 72, line after equation (3.28) the recurrent terms "Sijkl" and "Cijkl" were deleted 